\title{
EDITORIAL
}

\section{Is Life Necessary?}

\author{
Jack Trevors
}

Published online: 14 February 2007

(C) Springer Science + Business Media B.V. 2007

The biodiversity found in our biosphere is immense and still not fully identified. Given the likely millions of species of microorganisms and other unidentified eukaryotic species, we can conclude there is still a significant paucity of knowledge on the total biodiversity of our common biosphere. However, none of these diverse live forms are necessary. To the best of my knowledge, life is not necessary anywhere in the universe. The origin of life was not necessary, evolution and diversification of life was not necessary and humans are not necessary in the universe. In fact, the universe can carry on expanding and maybe even collapse at some time, all in the absence of any life forms. Our presence in the universe may therefore seems rather unimportant and insignificant. From a philosophical and scientific perspective this may be an accurate assessment, even though we do not know our future destiny and the life span of our emerging technological civilization. Some may disagree with me from a religious perspective.

On a more practical level, humans do think they are necessary in the universe. We want to enjoy our lives, our loved ones, relatives and friends. And most people I know certainly want to better humanity on a global scale. As humans we are faced with the realization that the universe can exist without us, but our minds/consciousness and humanity tells us that we value the sanctity of life and our common biosphere on the Earth. Let us seek global solutions to infectious diseases and pollution that are so devastating to life. We can turn our focus to the health and well-being of life forms in our biosphere as it is all we have at this time in our civilization.

J. Trevors $(\square)$

Department of Environmental Biology,

University of Guelph, Rm 3220 Bovey Bldg,

Guelph, ON N1G 2W1, Canada

e-mail: jtrevors@uoguelph.ca 